\title{
Descoloração de Corantes Alimentícios, Amarelo Crepúsculo e Azul Brilhante, Utilizando Radiação Ultravioleta
}

\author{
Fernando P. de Sá \& Liliane M. Nunes
}

Amarelo crepúsculo e azul brilhante são corantes sintéticos utilizados nas indústrias de alimentos e também fontes importantes de poluição da água, pois podem conter grandes concentrações de matéria orgânica e intensa coloração. Neste trabalho, foi estudada a cinética de descoloração das soluções por radiação ultravioleta. Os resultados mostraram a possibilidade de tratamento desses corantes, usando apenas ultravioleta. O processo fotoquímico apresentou resultados satisfatórios, com redução da DQO de $77,4 \%$ e $81,3 \%$ para o amarelo crepúsculo e azul brilhante, respectivamente. Os estudos cinéticos revelaram que a descoloração segue cinética de primeira ordem em relação à concentração de corantes.

Palavras-chave: Descoloração, Corantes Alimentícios, Ultravioleta.

Sunset Yellow and Brilliant Blue are synthetic dyes used in food industries and also important sources of water pollution because they can contain large concentrations of organic matter and have intense color. In this study, the discoloration kinetics of dyes was investigated by ultraviolet radiation. The results showed the possibility of treatment of these dyes, using only ultraviolet. The photochemical process presented satisfactory results, with reduction of DQO of $77,4 \%$ and $81,3 \%$ for the Sunset Yellow FCF and Brilliant Blue FCF, respectively. Kinetic investigations revealed that the discoloration follows first order kinetic with respect to the dyes concentration.

Keywords: discoloration; food colors; ultraviolet. 


\section{Introdução}

Economizar água para salvar o planeta e tornar o futuro da humanidade seguro é o slogan que vem sendo adotado no mundo todo como consequência das mudanças ambientais decorrentes da ação humana. O problema da poluição ambiental passou a ser um assunto de interesse público, visto que este tem se agravado ao longo dos anos, colocando em risco a saúde da comunidade, comprometendo as gerações futuras ${ }^{1}$. Assim, a poluição ambiental é identificada como um dos maiores problemas da sociedade moderna. A disponibilidade de água doce na natureza é limitada pelo alto custo de obtenção nas formas menos convencionais, como é o caso das águas subterrâneas. Portanto, é preciso dar maior prioridade à preservação, ao controle e à utilização racional das águas doces superficiais.

O colorido é o indicador mais inequívoco da poluição da água. Corantes sintéticos representam um grupo relativamente grande de produtos químicos orgânicos que são encontrados em praticamente todas as esferas da nossa vida diária. Estima-se que mais de 700.000 toneladas de 10.000 diferentes tipos de corantes são produzidas anualmente no mundo, sendo que aproximadamente 70.000 ton/ano são descartadas pelas indústrias ${ }^{1,2}$.

As indústrias alimentícias e de bebidas utilizam grande quantidade de corantes com a finalidade de reter a aparência do material original e tornar os produtos mais atrativos $^{3,4,5}$. Os efluentes descartados por essas indústrias são uma importante fonte de poluição dos corpos d'água. Quando descarregado em córregos, estes efluentes reduzem a transmissão da luz solar e podem provocar alterações na biota aquática (particularmente perto do ponto de lançamento $)^{6,7}$.

O problema da remoção da cor em efluentes coloridos tem encorajado a busca de várias formas de tratamento, tais como adsorção, coagulação/floculação, biológico, etc ${ }^{8 ;}$ 9; 10. Embora, em vários destes processos, o que ocorre é apenas a transferência de fase do poluente, necessitando de pós-tratamento dos resíduos sólidos gerados ou regeneração do material adsorvente. Consequentemente, existe uma necessidade imediata de desenvolvimento e utilização de processos que realmente possam degradar, ou preferencialmente mineralizar (conversão dos poluentes orgânicos em $\mathrm{CO}_{2}, \mathrm{H}_{2} \mathrm{O}$ e outros minerais) as espécies poluentes e, assim, garantir a qualidade de nossos recursos hídricos ${ }^{11}$.

A aplicação dos processos oxidativos avançados (POAs) no tratamento de compostos poluentes vem sendo apresentado como um dos mais eficazes na degradação e mineralização de corantes na degradação de compostos orgânicos e medicamentos ${ }^{6 ; 12 ; 13}$. Os POAs podem ser divididos em dois grupos principais: aqueles que envolvem reações homogêneas - utilizando $\mathrm{H}_{2} \mathrm{O}_{2}, \mathrm{O}_{3}$ e/ ou radiação ultravioleta (UV) e outros que envolvem reações heterogêneas - utilizando óxidos ou metais fotoativos14. Na fotólise direta com radiação ultravioleta, a luz é a única fonte capaz de produzir a destruição do poluente. Este trabalho visa avaliar a eficiência da radiação UV, na ausência de catalisadores, na descoloração de corantes alimentícios amarelo crepúsculo FCF (CI 15985) e azul brilhante FCF (CI 42090).

\section{Experimental}

\section{MATERIAIS}

Os corantes alimentícios amarelo crepúsculo e azul brilhante, Figura 1, foram gentilmente cedidos por Importadora Brastókio, sendo utilizados sem tratamento prévio.

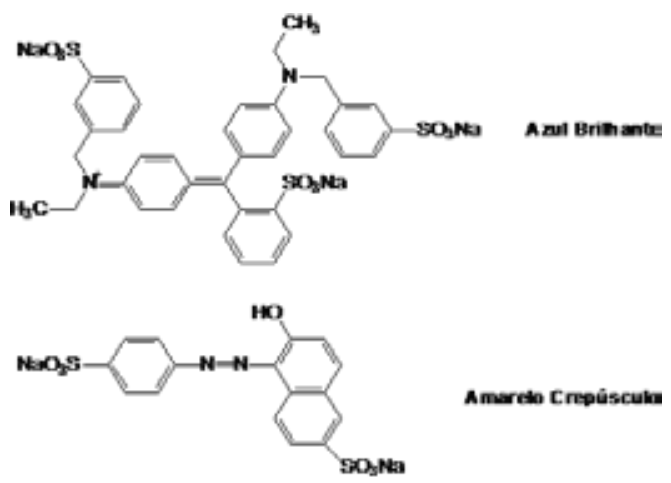

Figura 1. Estrutura molecular dos corantes Amarelo Crepúsculo e Azul Brilhante. 


\section{EXPERIMENTOS DE FOTODESCOLORAÇÃO}

A avaliação da descoloração das soluções foi realizada num aparato construído pelos autores deste trabalho, sendo constituído por uma célula cilíndrica de vidro (capaz de processar $400 \mathrm{~mL}$ de solução), um tubo de quartzo, no interior do qual foi colocado o filamento de uma lâmpada de vapor de mercúrio de $250 \mathrm{~W}$ (cuja cobertura de vidro foi previamente removida) e cilindro metálico para revestimento do reator, Figura 2. Para evitar um maior aquecimento, foi introduzido banho de gelo no interior do cilindro metálico, além de colocar todo este aparato no interior de uma capela, de modo a minimizar os efeitos da temperatura interna.

Os experimentos foram realizados em função do tempo de irradiação, utilizando-se $400 \mathrm{~mL}$ das soluções aquosas nas concentrações de 20 e $50 \mathrm{mg} / \mathrm{L}$, sem controle de $\mathrm{pH}$, sob agitação magnética. Em intervalos de tempo pré-estabelecidos, alíquotas da solução foram coletadas para posterior análise da concentração por espectroscopia UV-Vis.

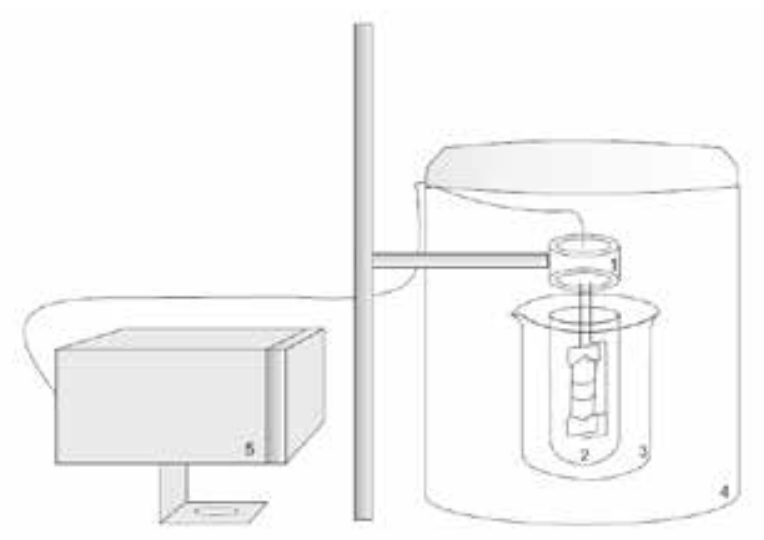

Figura 2: Aparato utilizado nos experimentos de fotodegradação com UV. (1) lâmpada vapor de mercúrio, (2) tubo de

Para uma análise quantitativa da velocidade de descoloração das soluções, sendo que alguns processos ocorrem de maneira mais rápida do que outros, foi feito um estudo da cinética das reações. A cinética de redução de compostos orgânicos em soluções aquosas pode ser de primeira ordem ou ordem zero, dependendo apenas de sua concentração $0^{15}$. A cinética será de ordem zero, se houver linearidade do gráfico da concentração em função do tempo e de primeira ordem se o logaritmo natural da concentração dos reagentes diminuir linearmente com o tempo, conforme Equação 1.

$$
\ln \mathrm{C} / \mathrm{C}_{0}=\mathrm{k}_{1} \mathrm{t}
$$

em que: $\mathrm{C}$ denota a concentração de corante em um tempo qualquer de amostragem; $\mathrm{CO}$ é a concentração inicial de corante; $\mathrm{k}_{1}$ é a constante cinética de velocidade de primeira ordem para o processo estudado.

\section{CARACTERIZAÇÃO}

O acompanhamento da descoloração das soluções foi realizadoporespectros copiaUV-VIS comespectrofotômetro digital (modelo SP-220, marca Biospectro), utilizando cubetas de quartzo de $5 \mathrm{~cm} 3$ (caminho óptico de $1 \mathrm{~cm}$ ), com o monitoramento das bandas 482 e $628 \mathrm{~nm}$ para amarelo crepúsculo e azul brilhante, respectivamente.

\section{Resultados e Discussão}

O comportamento da descoloração (tratamento fotolítico) dos corantes alimentícios, nas concentrações de 20 e $50 \mathrm{mg} / \mathrm{L}$, pode ser visto nas Figuras 3 e 4. Observa-se perfil semelhante, independentemente da concentração ou do corante, havendo considerável diminuição da coloração das soluções no decorrer do tempo de irradiação, sendo que este processo ocorre mais rapidamente para o amarelo crepúsculo. Resultados diferentes foram observados por Marmitti, Pirotta e Stulp $(2010)^{11}$ nos estudos de fotólise direta dos corantes amarelo crepúsculo, azul brilhante, amarelo tartrazina, azul indigotina, eritrosina e amaranto, em que os pesquisadores observaram alterações na coloração das soluções, somente, quando a irradiação ocorreu na presença de catalisadores. 


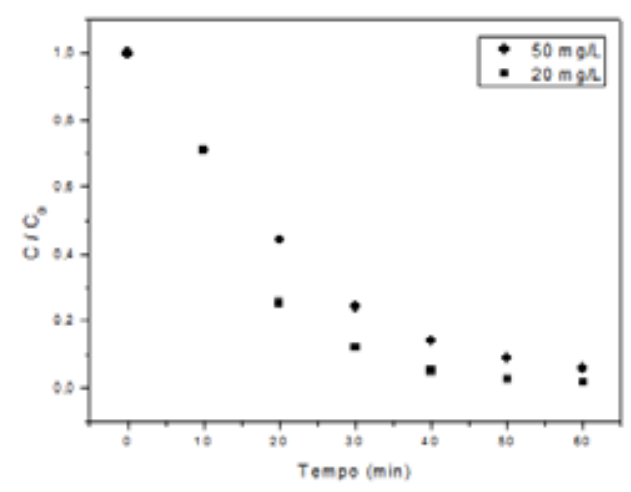

Figura 3: Descoloração das soluções ( 50 e $20 \mathrm{mg} / \mathrm{L}$ ) do corante amarelo crepúsculo versus tempo de irradiação.

Em 60 minutos de irradiação, a coloração da solução do amarelo crepúsculo $20 \mathrm{mg} / \mathrm{L}$ foi reduzida em $98 \%$, enquanto que a solução $50 \mathrm{mg} / \mathrm{L}$ reduziu $89 \%$. Por outro lado, para o corante azul brilhante, percentuais de $97 \%$ para $20 \mathrm{mg} / \mathrm{L}$ e $92 \%$ para $50 \mathrm{mg} / \mathrm{L}$, só são alcançados em 210 minutos. No entanto, nos primeiros 60 minutos, é observada uma redução de, apenas, $60 \%$. Este fato pode ser explicado pela diferença nas estruturas moleculares dos dois corantes, tendo em vista que a quebra das ligações no corante azul brilhante acontece mais lentamente por possuir uma estrutura molecular mais complexa, Figura 1. De acordo com Khataee et al. $(2010)^{16}$, o grupo azo, é susceptível à fotodegradação, o que faz com que o corante amarelo crepúsculo, seja mais facilmente degradado.

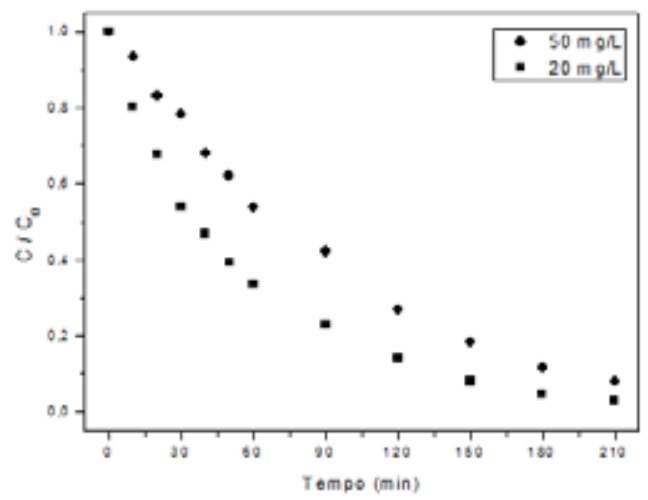

Figura 4: Descoloração das soluções (50 e $20 \mathrm{mg} / \mathrm{L})$ do corante azul brilhante versus tempo de irradiação.
O perfil das curvas ilustrado pelas Figuras 5 e 6, obtidas a partir da regressão linear das curvas experimentais da descoloração, sugerem uma cinética de primeira ordem, pois a diminuição no logaritmo da concentração ocorre linearmente com o tempo. Estes resultados são semelhantes aos apresentados na literatura para os estudos de fotodegradação de diversos corantes. ${ }^{1,19}$

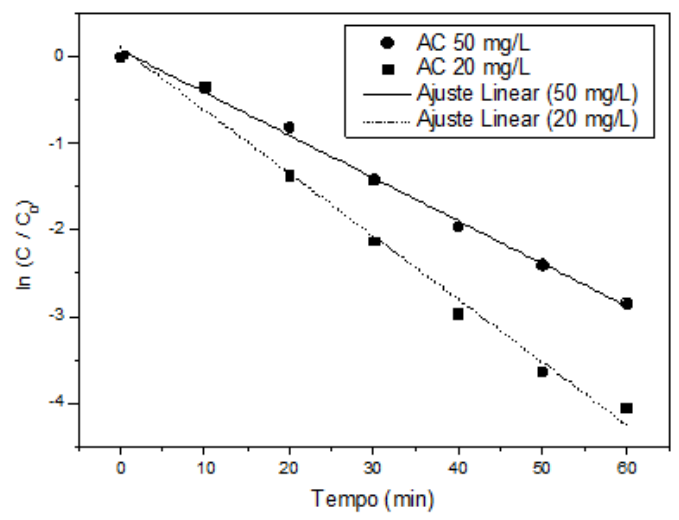

Figura 5: Curva de primeira ordem para a descoloração das soluções (50 e $20 \mathrm{mg} / \mathrm{L}$ ) do corante amarelo crepúsculo versus tempo de irradiação.

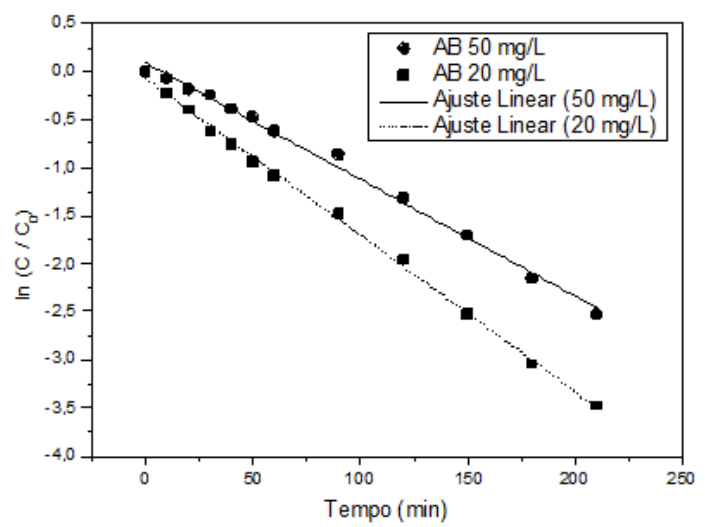

Figura 6: Curva de primeira ordem para descoloração das soluções (50 e $20 \mathrm{mg} / \mathrm{L}$ ) do corante azul brilhante versus tempo de irradiação.

Os parâmetros cinéticos, calculados a partir das Figuras 5 e 6, estão apresentados na Tabela 1 . Os valores de k1 comprovam quantitativamente o que é observado nas Figuras 3 e 4, ou seja, a descoloração das soluções acontece mais 
favoravelmente nas concentrações menores, independente do tipo de corante. Sendo estes valores de k1 compatíveis aos obtidos por Catanho et al. (2006) ${ }^{6}$ no estudo de degradação de corantes têxteis com tratamento fotoeletroquímico.

Tabela 1. Parâmetros cinéticos calculados para os corantes

\begin{tabular}{|c|c|c|c|}
\hline Corante & $\begin{array}{c}\text { Concentração } \\
(\mathbf{m g} / \mathbf{L})\end{array}$ & $\mathbf{k 1}$ (min-1) & $\mathbf{R 2}$ \\
\hline $\begin{array}{c}\text { Amarelo } \\
\text { crepúsculo }\end{array}$ & 20 & 0,032 & 0,987 \\
\hline Azul brilhante & 20 & 0,016 & 0,998 \\
\hline $\begin{array}{c}\text { Amarelo } \\
\text { crepúsculo }\end{array}$ & 50 & 0,021 & 0,995 \\
\hline Azul brilhante & 50 & 0,012 & 0,994 \\
\hline
\end{tabular}

As Figuras 7 e 8 ilustram os espectros de absorbância para as amostras dos corantes amarelo crepúsculo e azul brilhante, respectivamente, durante o processo de descoloração. Observa-se um decréscimo das bandas de absorção, características destes corantes $(\lambda=482 \mathrm{~nm}$ e 628 $\mathrm{nm})$ no decorrer do tempo de irradiação. Segundo a literatura, este comportamento está associado ao rompimento das ligações $(-\mathrm{N}=\mathrm{N}-$ e $\mathrm{C}=\mathrm{N}-)$, resultando na descoloração das soluções $16,17,18$. Durante todo o processo, não houve o surgimento de nenhuma nova banda de absorção na região do visível ou mesmo na ultravioleta, resultado semelhante ao observado por Khataee et al. (2009) ${ }^{19}$ no estudo de fotodegradação do corante azul brilhante, com uso de $\mathrm{TiO} 2$ como catalisador, o que mostra que o processo de descoloração aconteceu sem a formação de subprodutos.

Por outro lado, de acordo com Salgado et al. (2009) ${ }^{20}$, na região de comprimento de onda $(\lambda=210 \mathrm{~nm})$ é esperada a absorção da maioria dos subprodutos formados na degradação dos compostos contendo grupos cromóforos tipo azo e índigo. Portanto, como é mostrado na Figura 7, não houve absorção nesta região durante todo $\mathrm{o}$ processo, indicando que a descoloração do corante amarelo crepúsculo, também, aconteceu sem a formação indesejada de subprodutos.

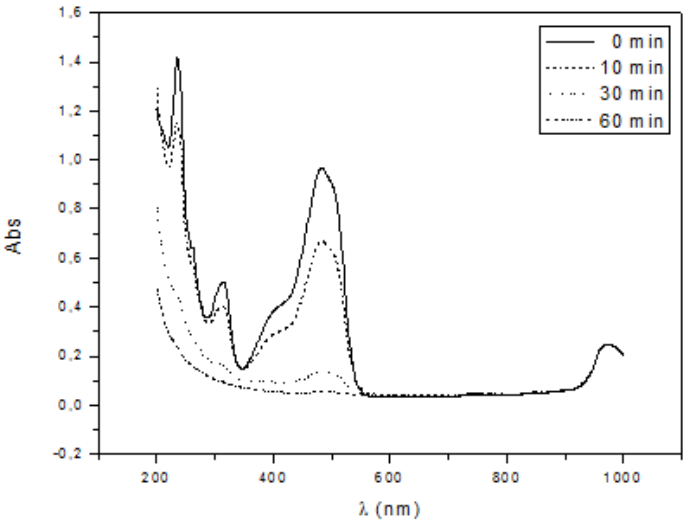

Figura 7: Espectro UV/Vis do corante amarelo crepúsculo (20 mg/L), em diferentes tempos de irradiação.

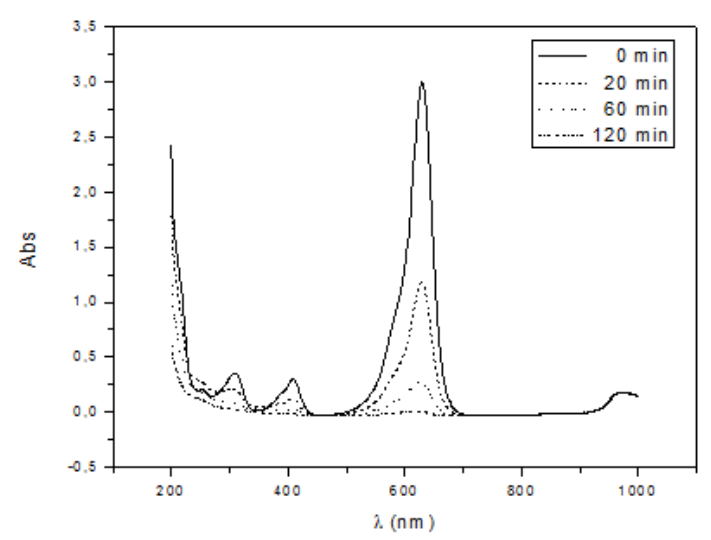

Figura 8: Espectro UV/Vis do corante azul brilhante ( $20 \mathrm{mg} / \mathrm{L})$, em diferentes tempos de irradiação.

\section{Conclusões}

Os resultados mostraram que existe a possibilidade de tratamento dos corantes alimentícios utilizando apenas radiação ultravioleta (tratamento fotolítico), não sendo necessária a adição de agente oxidante para o processo de descoloração. A eficiência, ao final deste processo, alcançou percentuais de $98 \%$ para o corante amarelo crepúsculo e 97 \% para azul brilhante, sendo a velocidade de descoloração muito maior para o corante amarelo crepúsculo. $\mathrm{O}$ estudo cinético mostrou que o clareamento das soluções segue um comportamento de primeira ordem, corroborando com os dados experimentais. 
Os espectros de absorbância para as amostras dos dois corantes mostraram uma diminuição das bandas de absorção características destes materiais no decorrer do tempo de irradiação. Também não foram observadas bandas de absorção relacionadas com a formação de subprodutos intermediários, comprovando que não apenas acontece a descoloração das soluções, como há indícios de uma degradação por completo dos compostos.

\section{Referências}

1. Gupta, V.; Suhas. J. Environ. Manage. 2009, 90, 2313.

2. Essawy, A. A.; Ali, A. E.-H.; Abdel-Mottaleb, M. J. Hazard. Mater. 2008, $157,547$.

3. Barros, F. A. R.; Stringheta, P. C.; Biotecnologia cienc. desenvolv. 2006, 36, 18.

4. Vidotti, E. C.; Rollemberg, M. C.; Quim. Nova. 2006, 29, 230

5. Abrantes, S. et al. Analytica. 2007, 27, 30.

6. Catanho, M.; Malpass, G. R. P.; Motheo, A. de J. Quím. Nova. 2006. 29(5), 983.

7. Srinivasan, A.; Viraraghavan, T. J. Environ. Manage. 2010, 91, 1915.

8. Purkait, M. et al. J. Hazard. Mater. 2007, 145, 287.

9. Santos, S. C.; Boaventura, R. A. Appl. Clay Sci. 2008, 42, 137.

10. Bhattacharyya, K. G.; Sarma, A. Dyes Pigm. 2003, 57, 211.

11. Marmitt, S.; Pirotta, L. V.; Stulp, S. Quim. Nova. 2010, 33(2), 384.

12. Cervantes, T. N. M.; Zaia, D. A. M.; Santana, H, Quim. Nova. 2009, $32(9), 2423$.

13. Souza, D. R. de et al. J. Photochem. Photobiol., A. 2006, 179, 269.

14. Wang, S. Dyes Pigm. 2008, 76, 714.

15. Silva, C. P. et al. Eng. Sanit. Ambiental. 2008, 13 (1), 73.

16. Khataee, A.; Kasiri, M. J. Mol. Catal. A: Chem. 2010, 328, 8.

17. Araujo, F. V. Da F.; Yokoyama, L.; Teixeira, L. A. C. Quim. Nova. 2006, $29(1), 11$.

18. Peralta-Hernández, J. et al. Dyes Pigm. 2008, 76, 656.

19. Khataee, A.; Vatanpour, V.; Ghadim, A. A. J. Hazard. Mater. 2009, $161,1225$.

20. Salgado, B. C. B. et al. Eng. Sanit. Ambiental. 2009, 14 (1),

\section{Fernando P. de Sá ${ }^{1, *} \&$ Liliane M. Nunes ${ }^{2}$}

${ }^{1}$ Instituto Federal de Educação, Ciência e Tecnologia de Goiás - IFG Avenida Universitária, s/n, Vale das Goiabeiras, CEP: 75400-000, Inhumas-GO, Brasil

${ }^{2}$ Instituto de Química, Universidade Federal de Goiás, 74001-970, Goiânia-GO, Brasil

*E-mail: fernandofpsa@gmail.com 